\title{
Generalized Fibonacci sequences in groupoids
}

\author{
Hee Sik Kim', J Neggers² and Keum Sook So ${ }^{3 *}$
}

"Correspondence:

ksso@hallym.ac.kr

${ }^{3}$ Department of Mathematics,

Hallym University, Chuncheon,

200-702, Korea

Full list of author information is

available at the end of the article

\begin{abstract}
In this paper, we introduce the notion of generalized Fibonacci sequences over a groupoid and discuss it in particular for the case where the groupoid contains idempotents and pre-idempotents. Using the notion of Smarandache-type $P$-algebra, we obtain several relations on groupoids which are derived from generalized Fibonacci sequences.

MSC: 11B39; 20N02; 06F35

Keywords: (generalized) Fibonacci sequences; $\left(L^{4}, L R L^{2}, R^{4}\right)$-groupoids; d/BCK-algebra; (pre-)idempotent; Smarandache disjoint
\end{abstract}

\section{Introduction}

Fibonacci-numbers have been studied in many different forms for centuries and the literature on the subject is consequently incredibly vast. Surveys and connections of the type just mentioned are provided in [1] and [2] for a very minimal set of examples of such texts, while in [3] an application (observation) concerns itself with the theory of a particular class of means which has apparently not been studied in the fashion done there by two of the authors of the present paper. Han et al. [4] studied a Fibonacci norm of positive integers, and they presented several conjectures and observations.

Given the usual Fibonacci-sequences $[1,2]$ and other sequences of this type, one is naturally interested in considering what may happen in more general circumstances. Thus, one may consider what happens if one replaces the (positive) integers by the modulo integer $n$ or what happens in even more general circumstances. The most general circumstance we will deal with in this paper is the situation where $(X, *)$ is actually a groupoid, i.e., the product operation $*$ is a binary operation, where we assume no restrictions $a$ priori. Han et al. [5] considered several properties of Fibonacci sequences in arbitrary groupoids.

The notion of $B C K$-algebras was introduced by Iséki and Imai in 1966. This notion originated from both set theory and classical and non-classical propositional calculi. The operation $*$ in $B C K$-algebras is an analogue of the set-theoretical difference. Nowadays, $B C K$-algebras have been studied by many authors and they have been applied to many branches of mathematics such as group theory, functional analysis, probability theory, topology and fuzzy theory $[6-8]$ and so on. We refer to $[9,10]$ for further information on $B C K / B C I$-algebras.

Let $(X, *)$ be a groupoid (or an algebra). Then $(X, *)$ is a Smarandache-type P-algebra if it contains a subalgebra $(Y, *)$, where $Y$ is non-trivial, i.e., $|Y| \geq 2$, or $Y$ contains at

\section{Springer}

C 2013 Kim et al.; licensee Springer. This is an Open Access article distributed under the terms of the Creative Commons Attribution License (http://creativecommons.org/licenses/by/2.0), which permits unrestricted use, distribution, and reproduction in any medium, provided the original work is properly cited. 
least two distinct elements, and $(Y, *)$ is itself of type $P$. Thus, we have Smarandachetype semigroups (the type $P$-algebra is a semigroup), Smarandache-type groups (the type $P$-algebra is a group), Smarandache-type Abelian groups (the type $P$-algebra is an Abelian group). Smarandache semigroup in the sense of Kandasamy is in fact a Smarandache-type group (see [11]). Smarandache-type groups are of course a larger class than Kandasamy's Smarandache semigroups since they may include non-associative algebras as well.

In this paper, we introduce the notion of generalized Fibonacci sequences over a groupoid and discuss it in particular for the case where the groupoid contains idempotents and pre-idempotents. Using the notion of Smarandache-type $P$-algebra, we obtain several relations on groupoids which are derived from generalized Fibonacci sequences.

\section{Preliminaries}

Given a sequence $\left\langle\varphi_{0}, \varphi_{1}, \ldots, \varphi_{n}, \ldots\right\rangle$ of elements of $X$, it is a left-*-Fibonacci sequence if $\varphi_{n+2}=\varphi_{n+1} * \varphi_{n}$ for $n \geq 0$, and a right-*-Fibonacci sequence if $\varphi_{n+2}=\varphi_{n} * \varphi_{n+1}$ for $n \geq 0$. Unless $(X, *)$ is commutative, i.e., $x * y=y * x$ for all $x, y \in X$, there is no reason to assume that left- $*$-Fibonacci sequences are right- $*$-Fibonacci sequences and conversely. We will begin with a collection of examples to note what, if anything, can be concluded about such sequences.

Example 2.1 Let $(X, *)$ be a left-zero-semigroup, i.e., $x * y:=x$ for any $x, y \in X$. Then $\varphi_{2}=$ $\varphi_{1} * \varphi_{0}=\varphi_{1}, \varphi_{3}=\varphi_{2} * \varphi_{1}=\varphi_{2}=\varphi_{1}, \varphi_{4}=\varphi_{3} * \varphi_{2}=\varphi_{3}=\varphi_{1}, \ldots$ for any $\varphi_{0}, \varphi_{1} \in X$. It follows that $\left\langle\varphi_{n}\right\rangle_{L}=\left\langle\varphi_{0}, \varphi_{1}, \varphi_{1}, \ldots\right\rangle$. Similarly, $\varphi_{2}=\varphi_{0} * \varphi_{1}=\varphi_{0}, \varphi_{3}=\varphi_{1} * \varphi_{2}=\varphi_{1}, \varphi_{4}=\varphi_{2} * \varphi_{3}=\varphi_{2}=\varphi_{0}$, $\ldots$ for any $\varphi_{0}, \varphi_{1} \in X$. It follows that $\left\langle\varphi_{n}\right\rangle_{R}=\left\langle\varphi_{0}, \varphi_{1}, \varphi_{0}, \varphi_{1}, \varphi_{0}, \varphi_{1}, \ldots\right\rangle$. In particular, if we let $\varphi_{0}:=0, \varphi_{1}:=1$, then $\left\langle\varphi_{n}\right\rangle_{L}=\langle 0,1,1,1,1, \ldots\rangle$ and $\left\langle\varphi_{n}\right\rangle_{R}=\langle 0,1,0,1,0,1, \ldots\rangle$.

Theorem 2.2 Let $\left\langle\varphi_{n}\right\rangle_{L}$ and $\left\langle\varphi_{n}\right\rangle_{R}$ be the left-*-Fibonacci and the right-*-Fibonacci sequences generated by $\varphi_{0}$ and $\varphi_{1}$. Then $\left\langle\varphi_{n}\right\rangle_{L}=\left\langle\varphi_{n}\right\rangle_{R}$ if and only if $\varphi_{n} *\left(\varphi_{n-1} * \varphi_{n}\right)=$ $\left(\varphi_{n} * \varphi_{n-1}\right) * \varphi_{n}$ for any $n \geq 1$.

A $d$-algebra [12] $(X, *, 0)$ is an algebra satisfying the following axioms: (D1) $x * x=0$ for all $x \in X$; (D2) $0 * x=0$ for all $x \in X$; (D3) $x * y=y * x=0$ if and only if $x=y$.

A $B C K$-algebra [13] is a $d$-algebra $X$ satisfying the following additional axioms:

(D4) $((x * y) *(x * z)) *(z * y)=0$,

(D5) $(x *(x * y)) * y=0$ for all $x, y, z \in X$.

Given algebra types $(X, *)\left(\right.$ type- $\left.P_{1}\right)$ and $(X, \circ)\left(\right.$ type- $\left.P_{2}\right)$, we will consider them to be Smarandache disjoint [11] if the following two conditions hold:

(i) If $(X, *)$ is a type- $P_{1}$-algebra with $|X|>1$, then it cannot be a Smarandache-type- $P_{2}$-algebra $(X, 0)$;

(ii) If $(X, \circ)$ is a type- $P_{2}$-algebra with $|X|>1$, then it cannot be a Smarandache-type- $P_{1}$-algebra $(X, *)$.

This condition does not exclude the existence of algebras $(X, \diamond)$ which are both Smarandache-type- $P_{1}$-algebras and Smarandache-type- $P_{2}$-algebras. It is known that semigroups and $d$-algebras are Smarandache disjoint [11].

It is known that if $(X, *, 0)$ is a $d$-algebra, then it cannot be a Smarandache-type semigroup, and if $(X, *)$ is a semigroup, then it cannot be a Smarandache-type $d$-algebra [11]. 


\section{Generalized Fibonacci sequences over $(X, *)$}

Let $Z_{2}[[X]]$ denote the power-series ring over the field $Z_{2}=\{0,1\}$. Given $p(x)=p_{0}+p_{1} x+$ $p_{2} x^{2}+\cdots \in Z_{2}[[X]]$, we associate a sequence $\bigwedge_{p(x)}=\left\{\lambda_{0}, \lambda_{1}, \lambda_{2}, \ldots\right\}$, where $\lambda_{i}=L$ if $p_{i}=0$ and $\lambda_{i}=R$ if $p_{i}=1$, which gives some information to construct a generalized Fibonacci sequence in a groupoid $(X, *)$.

Given a groupoid $(X, *)$ and a power-series $p(x) \in Z_{2}[[X]]$, if $a, b \in X$, we construct a sequence as follows:

$$
[a, b]_{p(x)}:=\left\{a, b, u_{0}, u_{1}, u_{2}, \ldots, u_{k}, \ldots\right\}
$$

where

$$
\begin{aligned}
& u_{0}:= \begin{cases}a * b & \text { if } \lambda_{0}=R, \\
b * a & \text { if } \lambda_{0}=L,\end{cases} \\
& u_{1}:=\left\{\begin{array}{ll}
b * u_{0} & \text { if } \lambda_{1}=R, \\
u_{0} * b & \text { if } \lambda_{1}=L
\end{array} \text { and } u_{k+2}:= \begin{cases}u_{k} * u_{k+1} & \text { if } \lambda_{k+2}=R, \\
u_{k+1} * u_{k} & \text { if } \lambda_{k+2}=L .\end{cases} \right.
\end{aligned}
$$

We call such a sequence $[a, b]_{p(x)}$ a $p(x)$-Fibonacci sequence over $(X, *)$ or a generalized Fibonacci sequence over $(X, *)$.

Example 3.1 Let $(X, *)$ be a groupoid and $a, b \in X$. If $p(x)=1+0 x+1 x^{2}+1 x^{3}+0 x^{4}+\cdots$ is a sequence in $Z_{2}[[X]]$, then we obtain $\bigwedge_{p(x)}=\{R, L, R, R, L, L, \ldots\}$ and its $p(x)$-Fibonacci sequence $[a, b]_{p(x)}$ can be denoted as follows: $[a, b]_{p(x)}:=\left\{a, b, u_{0}, u_{1}, u_{2}, \ldots\right\}$, where $u_{0}=$ $a * b, u_{1}=u_{0} * b=(a * b) * b, u_{2}=u_{0} * u_{1}=(a * b) *[(a * b) * b], u_{3}=u_{1} * u_{2}=[(a * b) * b] *$ $\{(a * b) *[(a * b) * b]\}, u_{4}=u_{3} * u_{2}=[[(a * b) * b] *\{(a * b) *[(a * b) * b]\}] *[(a * b) *[(a * b) * b]]$, $\ldots$

Example 3.2 (a) Let $(X, *)$ be a right-zero semigroup and let $p_{0}(x):=0+0 x^{1}+0 x^{2}+0 x^{3}+$ $\cdots$. Then $\bigwedge_{p_{0}(x)}=\{L, L, L, \ldots\}$ and hence $[a, b]_{p_{0}(x)}=\{a, b, b * a=a,(b * a) * b=b,[(b * a) *$ $b] *(b * a)=a, \ldots\}=\{a, b, a, b, a, b, \ldots\}$ for any $a, b \in X$.

(b) Let $(X, *)$ be a left-zero semigroup and let $p_{1}(x):=1+x+x^{2}+x^{3}+\cdots$. Then $\bigwedge_{p_{1}(x)}=$ $\{R, R, R, \ldots\}$ and hence $[a, b]_{p_{1}(x)}=\{a, b, a, b, a, b, \ldots\}$ for any $a, b \in X$.

Example 3.3 If we let $S(x):=x+x^{3}+x^{5}+\cdots$ and $C(x):=1+x^{2}+x^{4}+x^{6}+\cdots$, then $S(x)+C(x)=p_{1}(x)$ and $S(x)=x C(x)$. It follows that $\bigwedge_{S(x)}=\{L, R, L, R, \ldots\}$ and $\bigwedge_{C(x)}=$ $\{R, L, R, L, R, \ldots\}$. Let $(X, *)$ be a groupoid and let $a, b \in X$. Then $[a, b]_{S(x)}=\{a, b, b * a, b *$ $(b * a),(b *(b * a)) *(b * a), \ldots\}$ and $[a, b]_{C(x)}=\{a, b, a * b,(a * b) * b,(a * b) *((a * b) * b), \ldots\}$.

Let $p(x) \in Z_{2}[[X]]$. A groupoid $(X, *)$ is said to be power-associative if, for any $a, b \in X$, there exists $k \in \mathbf{Z}$ such that the generalized Fibonacci sequence $[a, b]_{p(x)}$ has $u_{k-2}=u_{k-1}=u$ for some $u \in X$.

Proposition 3.4 Let $p(x) \in Z_{2}[[X]]$. Let $(X, *)$ be a groupoid havingan identity e, i.e., $x * e=$ $x=e * x$ for all $x \in X$. Then $(X, *)$ is power-associative if, for any $a, b \in X,[a, b]_{p(x)}$ contains $e$. 
Proof If $[a, b]_{p(x)}$ contains $e$, then there exists an $u \in X$ such that $[a, b]_{p(x)}$ has $\ldots, u, e, \ldots$ Since $[a, b]_{p(x)}$ is a generalized Fibonacci sequence, it contains $\ldots, u, e, u * e=e * u=u, e *$ $u=u * e=u, \ldots$, proving that $(X, *)$ is power-associative.

Let $(X, *)$ be a semigroup and let $x \in X$. We denote $x^{2}:=x * x$, and $x^{n+1}:=x^{n} * x=x * x^{n}$, where $n$ is a natural number.

Theorem 3.5 Let $p(x) \in Z_{2}[[X]]$. Let $(X, *)$ be a semigroup and let $a, b \in X$. If it is powerassociative, then $[a, b]_{p(x)}$ contains a subsequence $\left\{u_{k}\right\}$ such that $u_{k+n}=u^{F_{n+3}}$ for some $u \in X$, where $F_{n}$ is the usual Fibonacci number.

Proof Given $a, b \in X$, since $(X, *)$ is power-associative, $[a, b]_{p(x)}$ contains an element $u$ such that $u_{k-2}=u_{k-1}=u$. It follows that either $u_{k}=u_{k-1} * u_{k-2}=u * u=u^{2}$ or $u_{k}=u_{k-2} * u_{k-1}=$ $u * u=u^{2}$. This shows that either $u_{k+1}=u_{k} * u_{k-1}=u^{2} * u=u^{3}$ or $u_{k+1}=u_{k-1} * u_{k}=u *$ $u^{2}=u^{3}$. In this fashion, we have $u_{k+2}=u^{5}, u_{k+3}=u^{8}=u^{F_{6}}, \ldots, u_{k+n}=u^{F_{n+3}}$.

Let $(X, *)$ be a groupoid having the following conditions:

(A) $x *(y * x)=x$,

(B) $(x * y) * y=x * y$ for all $x, y \in X$.

Given $p_{1}(x)=\sum_{n=0}^{\infty} x^{n}$, for any $a, b \in X$, a generalized Fibonacci sequence $[a, b]_{p_{1}(x)}$ has the following periodic sequence:

$$
\begin{aligned}
{[a, b]_{p_{1}(x)} } & =\{a, b, a * b, b *(a * b),(a * b) *(b *(a * b)), \ldots\} \\
& =\{a, b, a * b, b, a * b, b, a * b, \ldots\} .
\end{aligned}
$$

We call this kind of a sequence periodic.

A $B C K$-algebra $(X, *, 0)$ is said to be implicative [13] if $x=x *(y * x)$ for all $x, y \in X$.

Proposition 3.6 Let $(X, *, 0)$ be an implicative BCK-algebra and let $a, b \in X$. Then the generalized Fibonacci sequence $[a, b]_{p_{1}(x)}$ is periodic.

Proof Every implicative $B C K$-algebra satisfies the conditions (A) and (B).

Proposition 3.7 Let $(X, *, 0)$ be a BCK-algebra and let $a, b \in X$. Then the generalized Fibonacci sequence $[a, b]_{p_{0}(x)}$ is of the form $\{a, b, b * a, 0,0,0, \ldots\}$.

Proof If $(X, *, 0)$ is a $B C K$-algebra, then $(x * y) * x=(x * x) * y=0 * y=0$ for all $x, y \in X$. It follows that $[a, b]_{p_{0}(x)}=\{a, b, b * a,(b * a) * b=0,0 *(b * a)=0,0 * 0=0,0,0, \ldots\}$.

\section{Idempotents and pre-idempotents}

A groupoid $(X, *)$ is said to have an exchange rule if $(x * y) * z=(x * z) * y$ for all $x, y, z \in X$.

Proposition 4.1 Let a groupoid $(X, *)$ have an exchange rule and let $b$ be an idempotent in $X$. Then $[a, b]_{p_{0}(x)}=\left\{a, b, b * a,(b * a)^{2},(b * a)^{3},(b * a)^{4}, \ldots\right\}$ for all $a \in X$.

Proof Given $a, b \in X$, since $(X, *)$ has an exchange rule, $(b * a) * b=(b * b) * a$. It follows from $b$ is an idempotent that $b * b=b$. This proves that $[a, b]_{p_{0}(x)}=\{a, b, b * a,(b * a) * b=$ $\left.(b * b) * a=b * a,(b * a) *(b * a)=(b * a)^{2},(b * a)^{3}, \ldots\right\}$. 
Corollary 4.2 Let a groupoid $(X, *)$ have an exchange rule and let $b$ and $b * a$ be idempotents in $X$. Then $[a, b]_{p_{0}(x)}=\{a, b, b * a, b * a, b * a, \ldots\}$.

Proof Straightforward.

A groupoid $(X, *)$ is said to have an opposite exchange rule if $x *(y * z)=y *(x * z)$ for all $x, y, z \in X$.

Proposition 4.3 Let a groupoid $(X, *)$ have an opposite exchange rule and let $b$ be an idempotent in $X$. Then $[a, b]_{p_{1}(x)}=\left\{a, b, a * b,(a * b)^{2},(a * b)^{3},(a * b)^{4}, \ldots\right\}$ for all $a \in X$.

Proof Given $a, b \in X$, since $(X, *)$ has an opposite exchange rule and $b$ is an idempotent in $X, b *(a * b)=a *(b * b)=a * b$ and $(a * b) *[b *(a * b)]=(a * b) *(a * b)=(a * b)^{2}$. This proves that $[a, b]_{p_{1}(x)}=\left\{a, b, a * b,(a * b)^{2},(a * b)^{3},(a * b)^{4}, \ldots\right\}$.

Proposition 4.4 Let $(X, *)$ be a groupoid having the condition (B). If $b *(b * a)$ is an idempotent in $X$ for some $a, b \in X$, then $[a, b]_{S(x)}=\{a, b, b * a, b *(b * a), b *(b * a), \ldots\}$.

Proof Given $a, b \in X$, since $(X, *)$ has the condition (B), we have $(b *(b * a)) *(b * a)=$ $b *(b * a)$ and $[b *(b * a)] *[(b *(b * a)) *(b * a)]=(b *(b * a)) *(b *(b * a))$. Since $b *(b * a)$ is an idempotent in $X$, it follows that $[a, b]_{S(x)}=\{a, b, b * a, b *(b * a), b *(b * a), \ldots\}$.

Proposition 4.5 Let $(X, *)$ be a groupoid having the condition (B). If $a * b$ is an idempotent in $X$ for some $a, b \in X$, then $[a, b]_{C(x)}=\{a, b, a * b, a * b, a * b, \ldots\}$.

Proof The proof is similar to Proposition 4.4.

A groupoid $(X, *)$ is said to be pre-idempotent if $x * y$ is an idempotent in $X$ for any $x, y \in X$. Note that if $(X, *)$ is an idempotent groupoid, then it is a pre-idempotent groupoid as well. If $(X, *, f)$ is a leftoid, i.e., $x * y:=f(x)$ for some map $f: X \rightarrow X$, then $f(f(x))=f(x)$ implies $(X, *, f)$ is a pre-idempotent groupoid.

Theorem 4.6 Let $(X, *)$ be a groupoid. Let $u \in X$ such that $[a, b]_{p(x)}=\{a, b, u, u, \ldots\}$ for any $a, b \in X$. Then $(X, *)$ is a pre-idempotent groupoid, and

(i) if $p(x)=0$, then $(b * a) * b=b * a$,

(ii) if $p(x)=1$, then $(a * b) * b=a * b$,

(iii) if $p(x)=x$, then $b *(b * a)=b * a$,

(iv) if $p(x)=1+x$, then $b *(a * b)=a * b$.

Proof (i) If $p(x)=0$, then $[a, b]_{p(x)}=\{a, b, b * a,(b * a) * b,((b * a) * b) *(b * a), \ldots\}$. It follows that $(b * a) * b=b * a$ and $b * a=((b * a) * b) *(b * a)=(b * a) *(b * a)$, proving that $(X, *)$ is a pre-idempotent groupoid with $(b * a) * b=b * a$.

(ii) If $p(x)=1$, then $[a, b]_{p(x)}=\{a, b,(a * b) * b,((a * b) * b) *(a * b), \ldots\}$. It follows that $(a * b) * b=a * b$ and $a * b=((a * b) * b) *(a * b)=(a * b) *(a * b)$, proving that $(X, *)$ is a pre-idempotent groupoid with $(a * b) * b=a * b$.

(iii) If $p(x)=1 x$, then $[a, b]_{p(x)}=\{a, b, b * a, b *(b * a),(b *(b * a)) *(b * a), \ldots\}$. It follows that $b *(b * a)=b * a$ and $b * a=(b *(b * a)) *(b * a)=(b * a) *(b * a)$, proving that $(X, *)$ is a pre-idempotent groupoid with $b *(b * a)=b * a$. 
(iv) If $p(x)=1+1 x$, then $[a, b]_{p(x)}=\{a, b, a * b, b *(a * b),(a * b) *(b *(a * b)), \ldots\}$. It follows that $b *(a * b)=a * b$ and $a * b=(a * b) *(b *(a * b))=(a * b) *(a * b)$, proving that $(X, *)$ is a pre-idempotent groupoid with $b *(a * b)=a * b$.

Remark Not every generalized Fibonacci sequence has such an element $u$ in $X$ as in Theorem 4.6 in the cases of $B C K$-algebras.

Example 4.7 Let $X:=\{0,1,2,3\}$ be a set with the following table:

\begin{tabular}{l|llll}
$*$ & 0 & 1 & 2 & 3 \\
\hline 0 & 0 & 0 & 0 & 0 \\
1 & 1 & 0 & 1 & 0 \\
2 & 2 & 2 & 0 & 0 \\
3 & 3 & 2 & 1 & 0
\end{tabular}

Then $(X, *, 0)$ is a $B C K$-algebra. If we let $p(x):=1+0 x$, then it is easy to see that $[1,2]_{p(x)}=$ $\{1,2,1,2,0,0, \ldots\},[2,3]_{p(x)}=\{2,3,0,0, \ldots\},[3,2]_{p(x)}=\{3,2,1,1,0,0, \ldots\}$.

A groupoid $(X, *)$ is said to be a semi-lattice if it is idempotent, commutative and associative.

Theorem 4.8 If $(X, *)$ is a semi-lattice and $p(x)=p_{0}+p_{1} x \in Z_{2}[[x]]$, then there exists an element $u \in X$ such that $[a, b]_{p(x)}=\{a, b, u, u, \ldots\}$ for any $a, b \in X$.

Proof If $(X, *)$ is a semi-lattice, then it is a pre-idempotent groupoid. Given $a, b \in X$, we have $(b * a) * b=(a * b) * b=a *(b * b)=a * b, b *(b * a)=(b * a) * a=b * a, b *(a * b)=$ $(b * a) * b=(a * b) * b=a *(b * b)=a * b,(a * b) * b=a *(b * b)=a * b$. If we take $u:=a * b$, then $[a, b]_{p(x)}=\{a, b, u, u, \ldots\}$ for any $a, b \in X$, in any case of Theorem 4.6.

\section{Smarandache disjointness}

Proposition 5.1 The class of d-algebras and the class of pre-idempotent groupoids are Smarandache disjoint.

Proof Let $(X, *, 0)$ be both a $d$-algebra and a pre-idempotent groupoid. Then $a * b=(a *$ $b) *(a * b)=0$ and $b * a=(b * a) *(b * a)=0$, by pre-idempotence and (D2), for any $a, b \in X$. By (D3), it follows that $a=b$, which proves that $|X|=1$.

Proposition 5.2 The class of groups and the class of pre-idempotent groupoids are Smarandache disjoint.

Proof Let $(X, *, 0)$ be both a group and a pre-idempotent groupoid. Then, for any $x \in X$, we have $x=x * e=(x * e) *(x * e)=x * x$. It follows that $e=x * x^{-1}=(x * x) * x^{-1}=x *\left(x * x^{-1}\right)=$ $x * e=x$, proving that $|X|=1$.

A groupoid $(X, *)$ is said to be an $L^{4}$-groupoid if, for all $a, b \in X$,

(L1) $((b * a) * b) *(b * a)=b * a$,

(L2) $(b * a) *((b * a) * b)=(b * a) * b$. 
Example 5.3 Let $X:=\mathbf{R}$ be the set of all real numbers. Define a map $\varphi: X \rightarrow X$ by $\varphi(x):=$ $\lceil x\rceil-\frac{1}{2}$, where $\lceil x\rceil$ is the ceiling function. Then $\varphi(\varphi(x))=\lceil\varphi(x)\rceil-\frac{1}{2}=\left\lceil\lceil x\rceil-\frac{1}{2}\right\rceil-\frac{1}{2}=$ $\lceil\varphi(x)\rceil-\frac{1}{2}=\varphi(x)$. Define a binary operation ' $*$ ' on $X$ by $x * y:=\varphi(y)$ for all $x, y \in X$. Then $(X, *)$ is an $L^{4}$-groupoid. In fact, for all $a, b \in X,((b * a) * b) *(b * a)=\varphi(b) * \varphi(a)=\varphi(\varphi(a))=$ $\varphi(a)=b * a$. Moreover, $(b * a) *((b * a) * b)=\varphi(a) * \varphi(b)=\varphi(\varphi(b))=\varphi(b)=(b * a) * b$.

Proposition 5.4 Let $(X, *)$ be a groupoid and let $p(x) \in Z_{2}[[x]]$ such that $p(x)=x^{4} q(x)$ for some $q(x) \in Z_{2}[[x]]$. If there exist $u, v \in X$ such that $[a, b]_{p(x)}=\left\{a, b, u, v, u, v, \alpha_{1}, \alpha_{2}, \ldots\right\}$, where $\alpha_{i} \in Z_{2}$ for any $a, b \in X$, then $(X, *)$ is an $L^{4}$-groupoid.

Proof Since $p(x)=x^{4} q(x)$, we have $\bigwedge_{p(x)}=\left\{L, L, L, L, \alpha_{1}, \alpha_{2}, \ldots\right\}$, where $\alpha_{i} \in\{L, R\}$. It follows that $[a, b]_{p(x)}=\{a, b, b * a,(b * a) * b,((b * a) * b) *(b * a),(((b * a) * b) *(b * a)) *((b * a) * b), \ldots\}$. This shows that $u=b * a, v=(b * a) * b$, and hence $((b * a) * b) *(b * a)=b * a$ and $(b * a) *((b * a) * b)=(b * a) * b$, proving the proposition.

Proposition 5.5 Every $L^{4}$-groupoid is pre-idempotent.

Proof Given $a, b \in X$, we have $(b * a) *(b * a)=((b * a) * b) *(b * a)=b * a$, proving the proposition.

Proposition 5.6 The class of $L^{4}$-groupoids and the class of groups are Smarandache disjoint.

Proof Let $(X, *)$ be both an $L^{4}$-groupoid and a group with identity $e$. Then $((b * a) * b) *$ $(b * a)=b * a$ for all $a, b \in X$. Since any group has the cancellation laws, we obtain $(b * a) * b=e$. If we apply this to (L2), then we have $(b * a) * e=e$. This means that $b * a=e$. It follows that $e=b * a=((b * a) * b) *(b * a)=(e * b) * e=b$, proving that $|X|=1$.

Proposition 5.7 The class of $L^{4}$-groupoids and the class of BCK-algebras are Smarandache disjoint.

Proof Let $(X, *)$ be both an $L^{4}$-groupoid and a $B C K$-algebra with a special element $0 \in X$. Given $a, b \in X$, we have $0=0 * a=(b * b) * a=(b * a) * b=(b * a) *((b * a) * b)=(b * a) * 0=$ $b * a$. Similarly, $a * b=0$. Since $X$ is a $B C K$-algebra, $a=b$ for all $a, b \in X$, proving that $X=\{0\}$.

Let $p(x) \in Z_{2}[[x]]$. A groupoid $(X, *)$ is said to be a Fibonacci semi-lattice if for any $a, b \in X$, there exists $u=u(a, b, p(x))$ in $X$ depending on $a, b, p(x)$ such that $[a, b]_{p(x)}=$ $\{a, b, u, u, \ldots\}$.

Note that every Fibonacci semi-lattice is a pre-idempotent groupoid satisfying one of the conditions $(b * a) * b=a * b, b *(b * a)=b * a, b *(a * b)=a * b,(a * b) * b=a * b$ separately (and simultaneously).

Proposition 5.8 Let $(X, *)$ be a groupoid and let $p(x) \in Z_{2}[[x]]$ such that $p(x)=x^{3} q(x)$ for some $q(x) \in Z_{2}[[x]]$ with $q_{0}=1$. Then $(X, *)$ is a Fibonacci semi-lattice.

Proof Since $p(x)=x^{3} q(x)$, where $q(x) \in Z_{2}[[x]]$ with $q_{0}=1$, we have $\bigwedge_{p(x)}=\left\{L, L, L, R, \alpha_{1}\right.$, $\left.\alpha_{2}, \ldots\right\}$, where $\alpha_{i} \in\{L, R\}$. If we let $u:=b * a, v:=(b * a) * b$, then $[a, b]_{p(x)}=\{a, b, b * a=$ 
$u,(b * a) * b=v,((b * a) * b) *(b * a)=v * u=u, \ldots\}$. It follows that $b * a=u=v * u=v=$ $(b * a) * b$. Hence $[a, b]_{p(x)}=\{a, b, b * a, b * a, \ldots\}$, proving the proposition.

A groupoid $(X, *)$ is said to be an $L R L^{2}$-groupoid if for all $a, b \in X$,

$\left(L R L^{2} 1\right) \quad(b *(b * a)) *(b * a)=b * a$,

$\left(L R L^{2} 2\right)(b * a) *(b *(b * a))=b *(b * a)$.

Proposition 5.9 Let $(X, *)$ be a groupoid and let $p(x) \in Z_{2}[[x]]$ such that $p_{0}=p_{2}=p_{3}=0$, $p_{1}=1$. Then $(X, *)$ is an $L R L^{2}$-groupoid.

Proof Since $p(x) \in Z_{2}[[x]]$ such that $p_{0}=p_{2}=p_{3}=0, p_{1}=1$, we have $\bigwedge_{p(x)}=\left\{L, R, L, L, \alpha_{1}\right.$, $\left.\alpha_{2}, \ldots\right\}$, where $\alpha_{i} \in\{L, R\}$. If we let $u:=b * a, v:=b *(b * a)$, then $[a, b]_{p(x)}=\{a, b, b * a=$ $u, b *(b * a)=v,(b *(b * a)) *(b * a)=u,[(b *(b * a)) *(b * a)] *[b *(b * a)], \ldots\}$. It follows that $(b *(b * a)) *(b * a)=b * a$ and $(b * a) *(b *(b * a))=b *(b * a)$, proving the proposition.

Proposition 5.10 The class of $L R L^{2}$-groupoids and the class of groups are Smarandache disjoint.

Proof Let $(X, *)$ be both an $L R L^{2}$-groupoid and a group with a special element $e \in X$. Given $a, b \in X$, we have $(b *(b * a)) *(b * a)=b * a$. Since every group has cancellation laws, we obtain $b *(b * a)=e$. It follows that $b * a=(b * a) * e=(b * a) *(b *(b * a))=b *(b * a)=e$, and hence $e=b *(b * a)=b * e=b$. This proves that $|X|=1$, proving the proposition.

A groupoid $(X, *)$ is said to be an $R^{4}$-groupoid if for all $a, b \in X$,

$\left(R^{4} 1\right)(a * b) *(b *(a * b))=a * b$,

$\left(R^{4} 2\right)(b *(a * b)) *(a * b)=b *(a * b)$.

Proposition 5.11 Let $(X, *)$ be a groupoid and let $p(x) \in Z_{2}[[x]]$ such that $p_{0}=p_{1}=p_{2}=$ $p_{3}=1$. Then $(X, *)$ is an $R^{4}$-groupoid.

Proof Since $p(x) \in Z_{2}[[x]]$ such that $p_{0}=p_{1}=p_{2}=p_{3}=1$, we have $\bigwedge_{p(x)}=\left\{R, R, R, R, \alpha_{1}\right.$, $\left.\alpha_{2}, \ldots\right\}$, where $\alpha_{i} \in\{L, R\}$. If we let $u:=a * b, v:=b *(a * b)$, then $[a, b]_{p(x)}=\{a, b, a * b=$ $u, b *(a * b)=v,(a * b) *(b *(a * b))=u,[b *(a * b)] *[(a * b) *(b *(a * b))], \ldots\}$. It follows that $a * b=u=u * v=(a * b) *(b *(a * b))$ and $b *(a * b)=v=v * u=(b *(a * b)) *(a * b)$, proving the proposition.

Theorem 5.12 The class of $R^{4}$-groupoids and the class of groups are Smarandache disjoint.

Proof Let $(X, *)$ be both an $R^{4}$-groupoid and a group with a special element $e \in X$. Given $a, b \in X$, we have $(a * b) *(b *(a * b))=a * b$. Since every group has cancellation laws, we obtain $b *(a * b)=e$. By applying $\left(R^{4} 2\right)$, we have $e *(a * b)=e$, i.e., $a * b=e$. By $\left(R^{4} 1\right)$, $b=e *(b * e)=e$. This proves that $|X|=1$, proving the proposition.

Proposition 5.13 Every implicative BCK-algebra is an $R^{4}$-groupoid.

Proof If $(X, *, 0)$ is an implicative $B C K$-algebra, then $a *(b * a)=a$ and $(a * b) * b=a * b$ for any $a, b \in X$. It follows immediately that $(X, *)$ is an $R^{4}$-groupoid. 
Remark The condition, implicativity, is important for a $B C K$-algebra to be an $R^{4}$ groupoid.

Example 5.14 Let $X:=\{0,1,2,3\}$ be a set with the following table:

\begin{tabular}{l|llll}
$*$ & 0 & 1 & 2 & 3 \\
\hline 0 & 0 & 0 & 0 & 0 \\
1 & 1 & 0 & 0 & 0 \\
2 & 2 & 1 & 0 & 1 \\
3 & 3 & 1 & 1 & 0
\end{tabular}

Then $(X, *, 0)$ is a $B C K$-algebra, but not implicative, since $1 *(3 * 1)=0 \neq 1$. Moreover, it is not a $R^{4}$-groupoid since $3 * 2=1 \neq 0=(3 * 2) *[2 *(3 * 2)]$.

Theorem 5.15 Every BCK-algebra $(X, *, 0)$ inherited from a poset $(X, \leq)$ is an $R^{4}$ groupoid.

Proof If $(X, *, 0)$ is a $B C K$-algebra $(X, *, 0)$ inherited from a poset $(X, \leq)$, then the operation ' $*$ ' is defined by

$$
x * y:= \begin{cases}0 & \text { if } x \leq y \\ x & \text { otherwise }\end{cases}
$$

Then the condition $\left(R^{4} 1\right)$ holds. In fact, given $x, y \in X$, if $x \leq y$, then $x * y=0$ and $y * x=y$. It follows that $(x * y) *(y *(x * y))=0 *(y * 0)=0=x * y$. If $y \leq x$, then $y * x=0$ and $x * y=x$. It follows that $(x * y) *(y *(x * y))=x *(y * x)=x * 0=x=x * y$. If $x$ and $y$ are incomparable, then $x * y=x$ and $y * x=y$. It follows that $(x * y) *(y *(x * y))=x *(y * x)=x * y$.

We claim that $\left(R^{4} 2\right)$ holds. Given $x, y \in X$, if $x \leq y$, then $x * y=0, y * x=y$. It follows that $(y *(x * y)) *(x * y)=(y * 0) * 0=y=y * 0=y *(x * y)$. If $y \leq x$, then $y * x=0, x * y=x$. It follows that $(y *(x * y)) *(x * y)=(y * x) * x=0 * x=0=y * x=y *(x * y)$. If $x$ and $y$ are incomparable, then $x * y=x$ and $y * x=y$. It follows that $(y *(x * y)) *(x * y)=(y * x) * x=$ $y * x=y *(x * y)$. This proves that the $B C K$-algebra $(X, *, 0)$ inherited from a poset $(X, \leq)$ is an $R^{4}$-groupoid.

Note that the $B C K$-algebra $(X, *, 0)$ inherited from a poset $(X, \leq)$ need not be an implicative $B C K$-algebra unless the poset $(X, \leq)$ is an antichain [14, Corollary 9].

Example 5.16 Let $(X, *)$ be a left-zero semigroup, i.e., $x * y=x$ for all $x, y \in X$. Then $(X, *)$ is an $R^{4}$-groupoid, but not a $B C K$-algebra. 


\section{Author details}

${ }^{1}$ Department of Mathematics, Research Institute for Natural Sciences, Hanyang University, Seoul, 133-791, Korea.

${ }^{2}$ Department of Mathematics, University of Alabama, Tuscaloosa, AL 35487-0350, USA. ${ }^{3}$ Department of Mathematics, Hallym University, Chuncheon, 200-702, Korea.

\section{Acknowledgements}

The authors are grateful for the referee's valuable suggestions and help.

Received: 14 September 2012 Accepted: 15 January 2013 Published: 30 January 2013

\section{References}

1. Atanassov, KT, Atanassova, V, Shannon, AG, Turner, JC: New Visual Perspectives on Fibonacci Numbers. World Scientific, Singapore (2002)

2. Dunlap, RA: The Golden Ratio and Fibonacci Numbers. World Scientific, Singapore (1997)

3. Kim, HS, Neggers, J: Fibonacci means and golden section mean. Comput. Math. Appl. 56, 228-232 (2008)

4. Han, JS, Kim, HS, Neggers, J: The Fibonacci norm of a positive integer $n$-observations and conjectures. Int. J. Number Theory 6, 371-385 (2010)

5. Han, JS, Kim, HS, Neggers, J: Fibonacci sequences in groupoids. Adv. Differ. Equ. 2012, 19 (2012). doi:10.1186/1687-1847-2012-19

6. Ma, X, Zhan, J, Jun, YB: Some kinds of $(\epsilon, \epsilon \vee q)$-interval-valued fuzzy ideals of BCl-algebras. Inf. Sci. 178, 3738-3754 (2008)

7. Ma, X, Zhan, J, Jun, YB: Some kinds of $\left(\epsilon_{\gamma}, \epsilon_{\gamma} \vee q_{\delta}\right)$-fuzzy ideals of BCl-algebras. Comput. Math. Appl. 61, 1005-1015 (2011)

8. Zhan, J, Jun, YB: On $(\epsilon, \epsilon \vee q)$-fuzzy ideals of BCl-algebras. Neural Comput. Appl. 20, 319-328 (2011)

9. Zhan, J, Tan, Z: m-maps of BCK-algebras. Sci. Math. Jpn. 56, 309-311 (2002)

10. Zhan, J, Tan, Z: On the kp-radical in BCl-algebras. Sci. Math. Jpn. 56, 309-311 (2002)

11. Allen, PJ, Kim, HS, Neggers, J: Smarandache disjoint in BCK/d-algebras. Sci. Math. Jpn. 61, 447-449 (2005)

12. Neggers, J, Kim, HS: On d-algebras. Math. Slovaca 49, 19-26 (1999)

13. Meng, J, Jun, YB: BCK-Algebras. Kyung Moon Sa Co., Seoul (1994)

14. Kim, JY, Jun, YB, Kim, HS: BCK-algebras inherited from the posets. Sci. Math. Jpn. 45, 119-123 (1997)

doi:10.1186/1687-1847-2013-26

Cite this article as: Kim et al.: Generalized Fibonacci sequences in groupoids. Advances in Difference Equations 2013 2013:26.

\section{Submit your manuscript to a SpringerOpen ${ }^{\ominus}$ journal and benefit from:}

- Convenient online submission

Rigorous peer review

- Immediate publication on acceptance

Open access: articles freely available online

- High visibility within the field

- Retaining the copyright to your article 